\title{
Long-term evolution and predictive factors of mild inflammatory bowel disease
}

\author{
C. Reenaers, C. Pirard, C. Vankemseke, P. Latour, J. Belaiche and E. Louis \\ Gastroenterology Department, University Hospital of Liège, Liège, Belgium
}

\begin{abstract}
Background Crohn's disease (CD) and ulcerative colitis (UC) are potentially progressive diseases. Few data are available on the prevalence and the factors associated with mild inflammatory bowel diseases (IBD). Aim Our aim was to assess the natural history of mild CD and mild UC and to identify predictive factors of mild
evolution over the long term.

Methods Retrospective study of IBD patients registered in the database of the university hospital CHU of Liège, Belgium. Mild CD was defined as an inflammatory luminal disease (no stricture, abdominal or perianal fistulae) requiring no immunomodulator (IM), anti-TNF and no surgery. Mild UC was defined as no requirement for IM, anti-TNF and no colectomy.

Results Four hundred and seventy-three CD and 189 UC were included (median follow-up: 13 and 11 years respectively). At 1 year, 147 patients had mild CD. At 5 years and the maximum follow-up, 56\% and $13 \%$ patients still had mild CD, respectively. At 1 year, 142 patients had mild UC. At 5 years and the maximum follow-up, $72 \%$ and $44 \%$ still had a mild UC, respectively. Factors associated with long-term mild CD and UC were older age at diagnosis and absence of corticosteroids in the first year. In UC proctitis location was associated with mild UC.
\end{abstract}

Conclusions In this cohort, $90 \%$ of CD patients and 3/4 of UC with mild disease at 1 year lost their mild disease status over time. An old age at diagnosis was predictive of the persistence of a mild CD and UC.

Keywords: Benign evolution; complication; mild IBD; predictive factor

\section{Introduction}

Crohn's disease (CD) and ulcerative colitis (UC) are potentially progressive diseases characterised by the occurrence of complications leading to irreversible tissue damages.[1,2] The aim of CD and UC treatments is not only to induce a corticosteroid-free remission, but also to achieve mucosal healing to change the evolution of the disease. Immunomodulators (IMs) and particularly anti-TNFs are effective to induce mucosal healing in inflammatory bowel diseases (IBD). Patients should be stratified according to their individual risk to be offered the best treatment strategy. Potential aggressive IBD must be detected at diagnosis to start effective treatment before the occurrence of complications. Predictive factors of severe and disabling disease have been identified.[3,4] However, intensive therapies are not required in every patient. Predicting patients with persistent mild disease would be useful to avoid overtreatment associated with side effects and high costs. Mild IBD has never been clearly defined. Few data are available for the prevalence and the factors associated with long-term mild IBD.[5] The primary objective of our study was to describe the evolution of mild IBD and to identify demographic and clinical factors at diagnosis associated with the persistence of a mild IBD over the time. The secondary objective was to describe the clinical significance and the factors associated with a mild IBD 1 year after the diagnosis.

\section{Patients and methods}

\section{Patients}

All patients with a complete follow-up reviewed in the IBD clinic of the CHU hospital of Liège in the last 3 years were considered for inclusion. The diagnosis of CD and UC was based on standard criteria.[6] Disease location and behaviour were determined according to Montreal classification.[7] A minimum follow-up of 5 years was required for inclusion. 
Published in: Scandinavian Journal of Gastroenterology (2016), vol. 51, n6, pp. 712-719 http://dx.doi.org/10.3109/00365521.2015.1128965

Status: Postprint (author's version)

\section{Definition of mild CD}

Mild CD was defined as an inflammatory luminal CD without strictures, abdominal or perianal fistulae, requiring no IM or anti-TNF and no surgery over the course of the disease.

\section{Definition of mild UC}

Mild UC was defined by the absence of IM, anti-TNF or surgery over the course of the disease.

\section{Study design}

Retrospective analysis of the evolution of mild CD and UC defined 1 year after the diagnosis. Demographic factors including age, sex, smoking habits were recorded at diagnosis. Disease characteristics including location and behaviour were recorded, 1 year after the diagnosis, 5 years after the diagnosis and at the maximum followup. Medical treatments, including corticosteroids, 5-aminosalicylates (5ASA), IM (thiopurines and methotrexate) and anti-TNF (infliximab, adalimumab, certolizumab), surgeries and hospitalisation for active IBD were recorded 1 year after the diagnosis, 5 years after the diagnosis and at the maximum follow-up.

\section{Definition of corticosteroids during the first year}

Only systemic corticosteroids were considered as treatment with corticosteroids during the first year. Topical corticosteroids, including suppositories and enema, oral budesonide and oral beclomethasone dipropionate were not considered as corticosteroid intake during the first year.

\section{Statistical analysis}

Descriptive statistics are expressed as mean (standard deviation) for continuous variables and as numbers (percentages) for qualitative variables. A logistic regression analysis was applied to test the association of demographic and clinical factors with mild IBD within the first year and with mild disease at maximum followup, the odds ratio and $95 \%$ confidence intervals are provided. Multivariate logistic regression analysis was done on variables for which $\mathrm{p}$ was less than 0.10 in univariate analysis. The loss of mild disease status is represented by a Kaplan-Meier curve. A Cox regression analysis was used to evaluate the persistence of mild CD or UC with respect to predictive factors. Results were considered to be significant at the 5\% confidence level $(p<0.05)$. Calculations were done in SAS version 9.3 (SAS Institute, Cary, NC).

\section{Results}

\section{Patients' recruitment and characteristics}

A total of 887 patients were screened for inclusion. Those without a definite diagnosis of CD or UC with incomplete data during follow-up or who did not meet the criteria of 5 years follow-up were excluded. The follow-up reported in the study describes all the events since the diagnosis of the patients till the last available follow-up after 5 years of evolution. We identified $473 \mathrm{CD}$ patients and $189 \mathrm{UC}$ patients suitable for the study. The median follow-up was 13 years (range: 8-22) and 11 years (range: 7-17) for CD and UC, respectively.

In the CD patients, $147 / 473$ (31\%) had a mild CD at 1 year according to the definition (Figure 1). Inflammatory, stricturing and penetrating CD 1 year after the diagnosis were observed in 329/473 (69\%), 89/473 (19\%) and $55 / 473(12 \%)$ of the patients, respectively. Perianal lesions 1 year after the diagnosis were reported in 119/473 patients $(25 \%)$. 5ASA, IM and anti-TNF were given during the first year after the diagnosis, respectively, in $319 / 473(67 \%), 104 / 473(20 \%)$ and 38/ $473(8 \%)$ of the patients. In patients with available data corticosteroids were administrated during the first year after diagnosis in $97 / 184$ patients $(53 \%)$. Hospitalisation and surgery for active CD during the first year occurred in 133/225 (59\%) and 89/473 (19\%) patients, respectively.

In the UC patients, $142 / 189$ (75\%) had a mild UC 1 year after the diagnosis according to the definition (Figure 1). 5ASA, IM and anti-TNF were given during the first year after the diagnosis, respectively, in 168/189 (89\%), $16 / 189(8 \%)$ and $3 / 189(2 \%)$ of the patients. Corticosteroids were administrated during the first year after the diagnosis in 37/96 (39\%); 21/73 (29\%) and 6/189 (3\%) patients were, respectively, hospitalised and operated for active UC during the first year after the diagnosis. Patients' characteristics are described in Table 1.

\section{Factors associated with mild IBD at 7 year}

In univariate analysis, mild CD 1 year after the diagnosis was associated with the absence of corticosteroids intake in the first year (66\% vs. $40 \%, p=0.008, \mathrm{OR}=2.31,95 \% \mathrm{CI}=1.25-4.29)$, and with 5ASA intake in the first year ( $96 \%$ vs. $86 \%, p=0.012$, OR $=3.52,95 \% \mathrm{CI}=1.33-9.32$ ). The multivariate analysis did not consider corticosteroids (data available in 184/473 patients) intake and hospitalisation (data available in 225/473 patients) because of incomplete data. No factor was significant in the multivariate analysis (Table 2). 
In UC, univariate analysis demonstrated that mild UC at 1 year was associated with the absence of corticosteroid intake in the first year (90\% vs. $24 \%, p<0.0001$, OR $=8.13,95 \% \mathrm{CI}=2.99-22.2)$, the absence of hospitalisation for active UC in the first year $(67 \%$ vs. $33 \%, p=0.0002$, $\mathrm{OR}=10.2,95 \% \mathrm{CI}=3.0-34.5)$, proctitis at 1 year $(p=$ 0.0002 , vs. rectosigmoiditis: $\mathrm{OR}=3.1,95 \% \mathrm{CI}=0.65-14.5$; vs. pancolitis: $\mathrm{OR}=12.5,95 \% \mathrm{CI}=2.7-58.8)$. The multivariate analysis did not consider corticosteroids intake (data available in 96/189 patients) and hospitalisation (data available in $73 / 189$ patients) because of incomplete data. In multivariate analysis, no factor was statistically predictive of mild UC at 1 year (Table 2).

Figure 1. Patients recruitment.

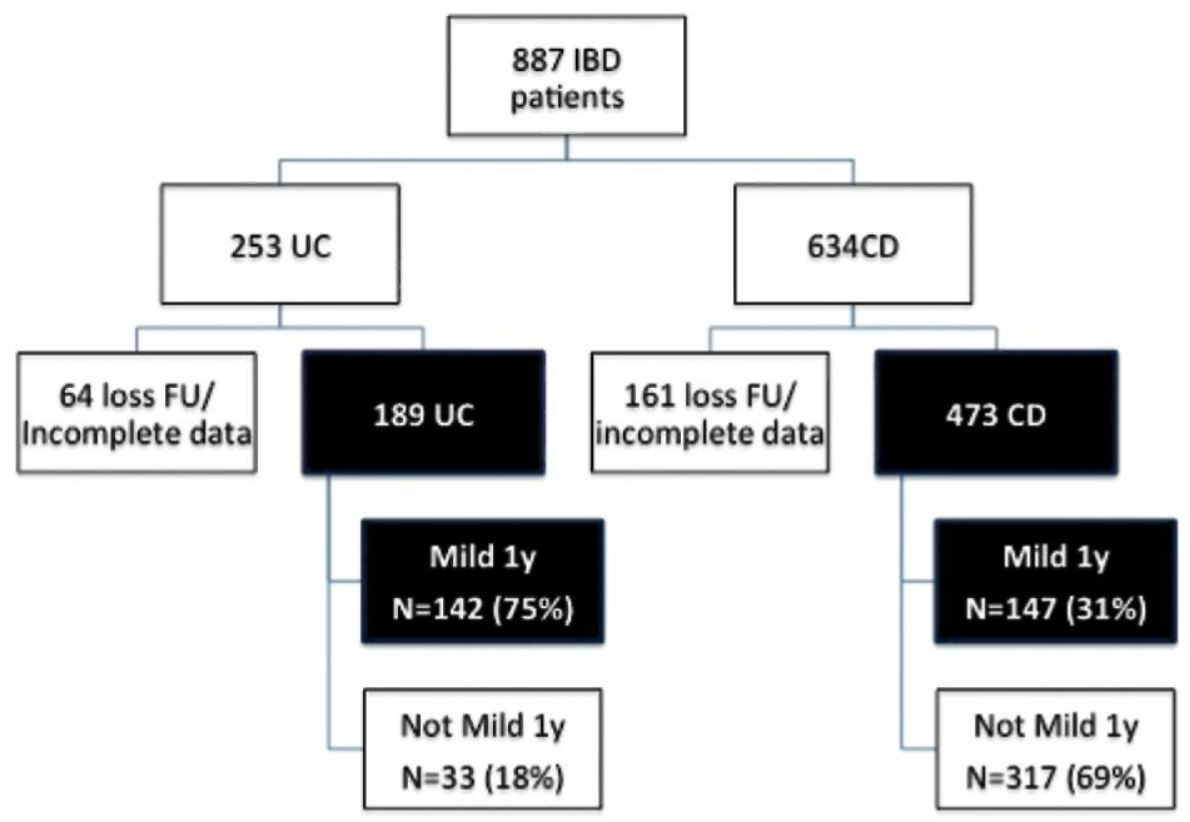

Table 1. Patients'characteristics.

\section{Patients characteristics}

Male gender

Age at diagnosis (median, range)

Disease duration in years (median, range)

Age at diagnosis (Montreal)

A2

A3

CD location

L1

L2

L3

$\mathrm{CD}$ behaviour at 1 year

B1

B2

B3

Perianal disease at 1 year

UC extend at 1 year

E1
E2
E3

Active smoker

Hospitalisation during the first year

Surgery during the first year

CS during the first year

\begin{tabular}{|c|c|}
\hline $\mathrm{CD}, n=473(\%)$ & $\mathrm{UC}, n=189(\%)$ \\
\hline $159(34)$ & $84(44)$ \\
\hline $24(7-69)$ & $33(10-78)$ \\
\hline $13(8-22)$ & $11(7-17)$ \\
\hline $47(10)$ & $10(5)$ \\
\hline $361(76)$ & $119(63)$ \\
\hline $65(14)$ & $60(32)$ \\
\hline \multicolumn{2}{|l|}{$198(42)$} \\
\hline \multicolumn{2}{|l|}{$80(17)$} \\
\hline \multicolumn{2}{|l|}{$192(41)$} \\
\hline \multicolumn{2}{|l|}{$329(69)$} \\
\hline \multicolumn{2}{|l|}{89 (19) } \\
\hline \multicolumn{2}{|l|}{$55(12)$} \\
\hline \multicolumn{2}{|l|}{$119(25)$} \\
\hline & $46(24)$ \\
\hline & $88(47)$ \\
\hline & $53(28)$ \\
\hline $163(34)$ & $24(12)$ \\
\hline $133 / 225(59)$ & $21 / 73(29)$ \\
\hline 89 (19) & $6(3)$ \\
\hline $97 / 184(53)$ & $37 / 96(39)$ \\
\hline
\end{tabular}


5ASA during the first year

Table 2. Factors associated with mild CD and UC 1 year after diagnosis.

\begin{tabular}{lll} 
Factors & $\boldsymbol{p}$ Value & OR (95\% CI) \\
\hline CD & & \\
5ASA intake* & $p=0.012$ & $3.52(1.33-9.32)$ \\
No CS intake & $p=0.008$ & $2.31(1.25-4.29)$ \\
UC & $p=0.001$ & $3.1(0.65-14.5)$ \\
Location* & $p=0.001$ & $12.5(2.7-58.8)$ \\
E1 vs. E2 & $p=0.0002$ & $10.2(3.0-34.5)$ \\
E1 vs. E3 & $p<0.0001$ & $8.13(2.99-22.2)$ \\
No hospitalisation* & & \\
No CS intake* & & \\
\hline
\end{tabular}

*univariate.

\section{Evolution of mild CD beyond 1 year}

Among the 147 patients having mild CD 1 year after the diagnosis, 83/147 (56\%) still had mild CD 5 years after the diagnosis (Figure 2). At 5 years, 11/147 (7\%), 9/147 (6\%) and 16/147 (11\%) developed a stricturing, penetrating and perianal CD, respectively. Abdominal surgery was performed in 20/147 patients (14\%). IM and anti-TNF were required in 36/147 (25\%) and 16/147 (11\%), respectively (Figure 3). At the maximum follow-up (median follow-up = 13 years), 19/147 (13\%) patients had a mild CD (Figure 2): 51/147 (28\%) developed strictures, 30/147 (17\%) developed fistulae, 55/147 (31\%) developed perianal lesions. Abdominal surgery was performed in 78/147 patients (43\%). IM and anti-TNF were required in 126/147 (70\%) and 90/147 (50\%), respectively (Figure 4). The median time for CD worsening (loss of mild disease status) is illustrated in Table 3. The Kaplan-Meier curve of the loss of mild CD is shown in Figure 5.

In univariate analysis, an older age at diagnosis either expressed as a continuous variable $(p=0.0005, \mathrm{OR}=$ 1.06, 95\% CI: 1.02-1.09) or as categories according to Montreal classification (A2 or A3 vs. A1: $p=0.011$, OR $=3.23$, 95\% CI: $1.31-7.94)$ was associated with long-term mild CD. The absence of corticosteroids intake during the first year after diagnosis was also associated with a mild CD in the long term $(p=0.0084$, OR $=3.33)$. In multivariate analysis, no factors remained associated with mild disease evolution.

Among patients with mild CD 1 year after the diagnosis factors associated with the persistence of mild CD over the time were analysed. In univariate analysis, the persistence of mild CD was associated with an older age at diagnosis either expressed as a continuous variable $(p=0.006, \mathrm{HR}=1.022,95 \% \mathrm{CI}: 1.01-1.04)$ or as categories according to Montreal classification (A2 or A3 vs. A1: $p=0.034$, HR=1.57, 95\% CI: 1.03-2.37) (Table 4). In multivariate analysis, no factors remained associated the persistence of mild CD in this sub-group.

Figure 2. Evolution of patients with mild IBD 1 year and 5 years after the diagnosis and at the maximum followup.

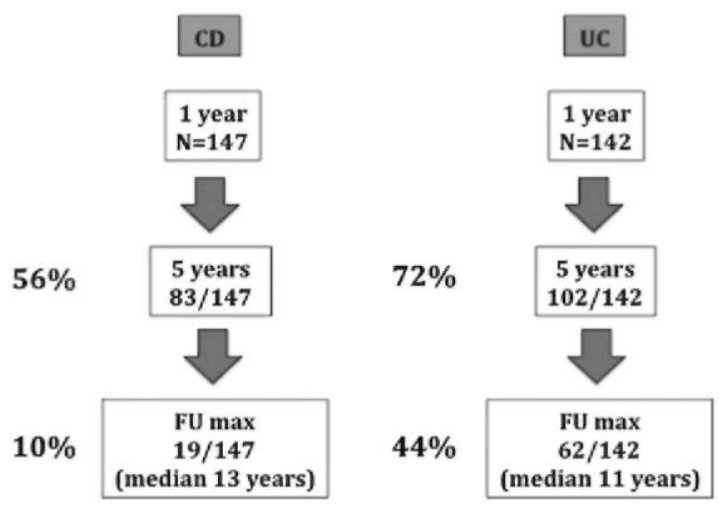


Figure 3. Mode of CD worsening 5 years after the diagnosis. The different events causing the loss of the mild character of the disease are represented in the figure. Five years after the diagnosis, few patients experienced events associated with tissue damage. The loss of the mild status was linked to the introduction of immunomodulators.

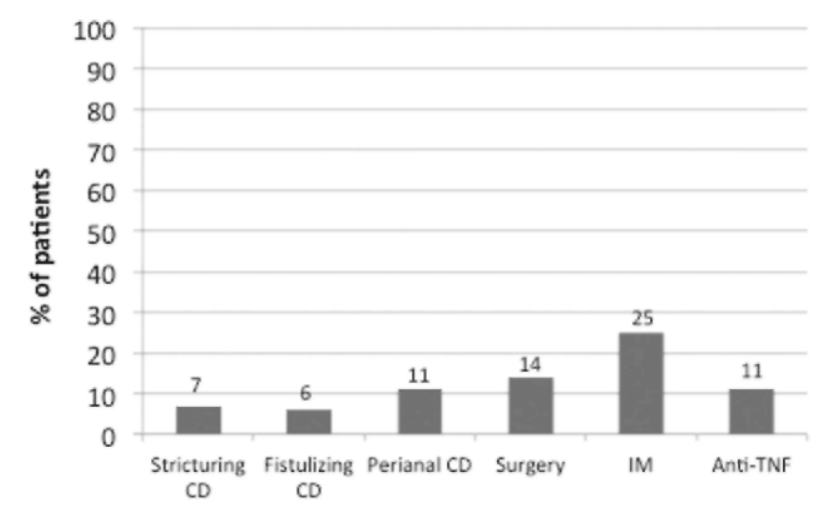

Figure 4. Mode of CD worsening at the maximum follow-up. The different events causing the loss of the mild character of the disease are represented in the figure. The complications leading to tissue damage increased compared to the evolution after 5 years. More than half of the patients were treated with IM and/or anti-TNF at the maximum follow-up.

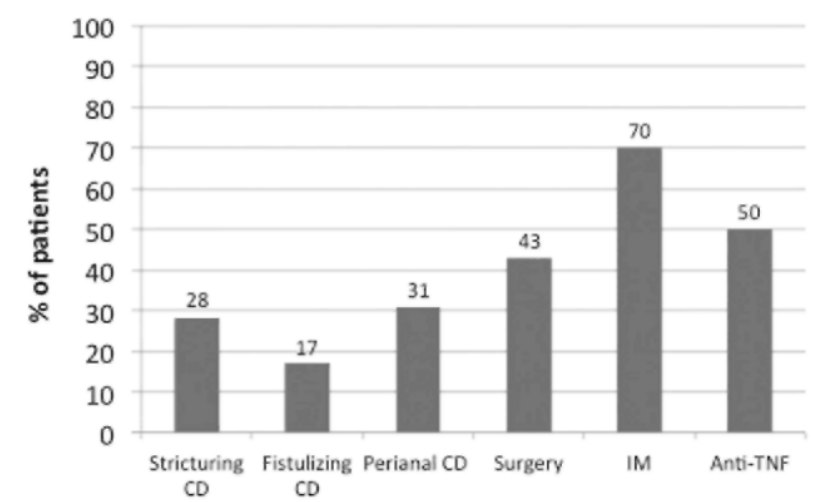

Figure 5. Survey with mild CD. Among the 147 patients with mild CD 1 year after the diagnosis $80 \%$ of the patients had mild CD 10 years after the diagnosis and $40 \% 20$ years after the diagnosis. After a longer period of time almost all the patients finally lost their mild status during the disease evolution.

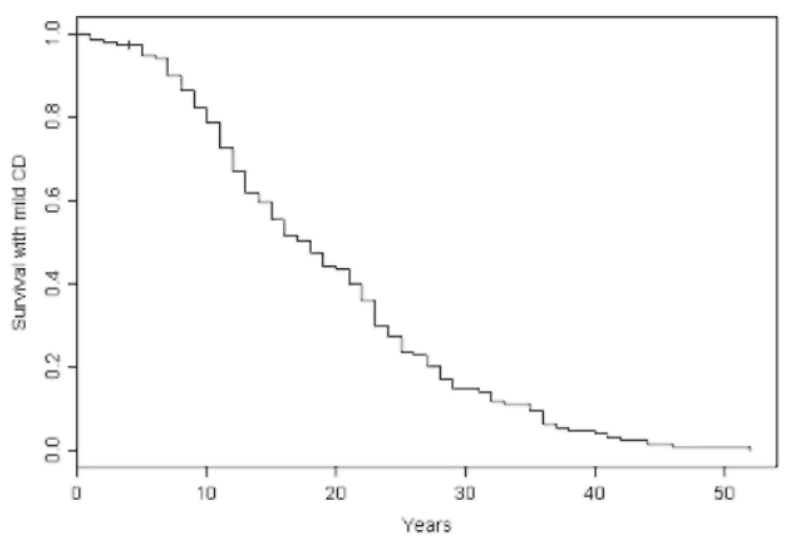


Table 3. Median time for loss of mild CD status.

Events inducing loss of mild CD status Months (IQR)

Stricturing disease $98(55-180)$

Fistulising disease

89 (34-174)

Perianal disease

89 (48-138)

Surgery

$91(48-169)$

IS

$72(23-132)$

Anti-TNF

89 (26-163)

Table 4. Factors associated with persistence of mild IBD in patients with mild IBD at 1 year.

\begin{tabular}{lcc} 
Factors & $\boldsymbol{p}$ Value & HR $(\mathbf{9 5 \%} \mathbf{C I})$ \\
\hline CD & & \\
$\quad$ Older age at diagnosis* & $p=0.006$ & $1.022(1.01-1.04)$ \\
$\quad$ Montreal A1 & $p=0.034$ & $1.57(1.03-2.37)$ \\
UC & & \\
$\quad$ Older age at diagnosis* & $p=0.006$ & $1.022(1.01-1.04)$ \\
\hline
\end{tabular}

*univariate.

Table 5. Median time from diagnosis to UC worsening.

\begin{tabular}{lc} 
Characteristic UC of worsening & Months (IQR) \\
\hline IS & $54(4-449)$ \\
Anti-TNF & $69(3-245)$ \\
Surgery & $125(24-480)$ \\
\hline
\end{tabular}

Figure 6. Mode of UC worsening 5 years after the diagnosis. The different events causing the loss of the mild character of the disease are represented in the figure. Only $2 \%$ of the patients required surgery. Immunomodulators and anti-TNF were prescribed in $23 \%$ and $9 \%$ of the patients, respectively.

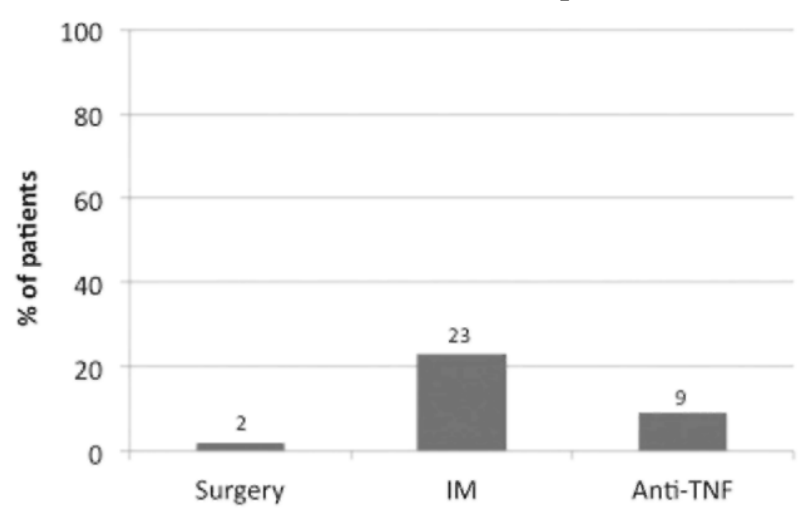

Figure 7. Mode of UC worsening at maximum follow-up. The different events causing the loss of the mild character of the disease are represented in the figure. Only $15 \%$ of the UC patients required surgery. Immunomodulators and anti-TNF were prescribed in $54 \%$ and $31 \%$ of the patients, respectively.

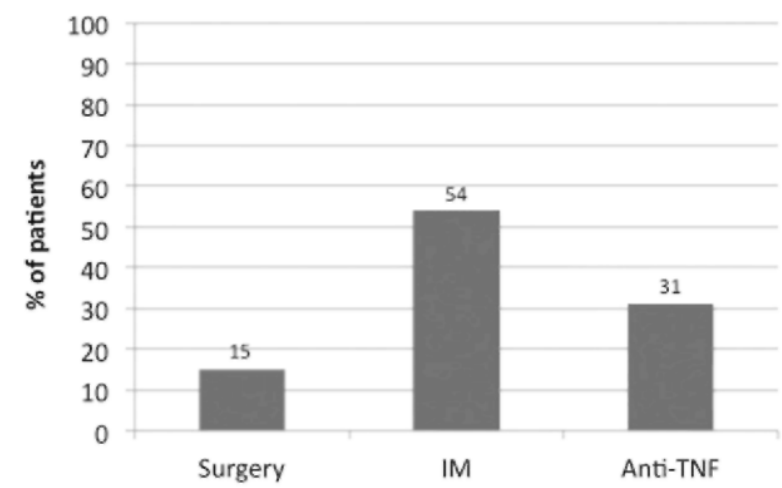


Figure 8. Survey with mild UC. Among the 142 patients with mild UC 1 year after the diagnosis still $80 \%$ of the patients had mild CD 10 years after the diagnosis and 40\% 20 years after the diagnosis. After a longer period of time there is a trend for a progressive loss of the mild character of the UC but the curve seems to reach a plateau.

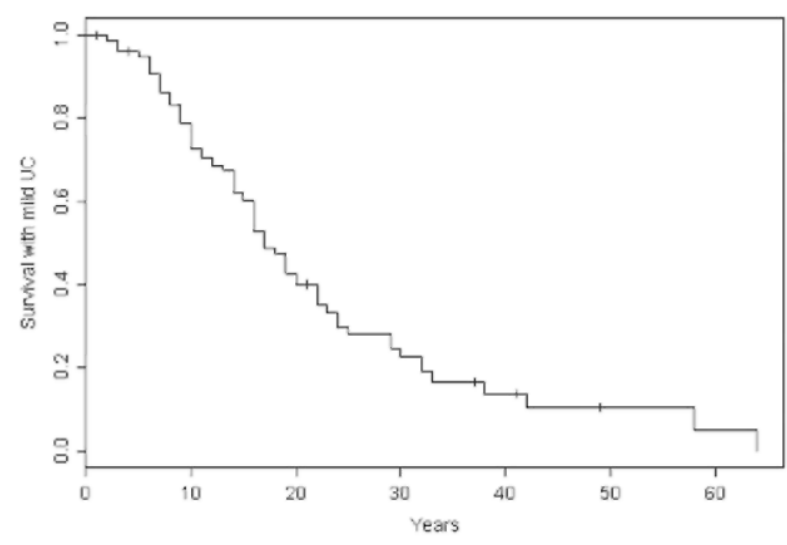

\section{Evolution of mild UC beyond 1 year}

Among the 142 patients having mild UC 1 year after the diagnosis, 102/142 (72\%) still had mild UC 5 years after the diagnosis (Figure 2). At 5 years, abdominal surgery was performed in 3/142 patients (2\%). IM and antiTNF were required in 33/142 (23\%) and 13/142 (9\%), respectively (Figure 6). At the maximum follow-up (median follow-up $=11$ years) 62/142 (44\%) patients had a mild UC (Figure 2). Surgery was performed in 21/142 patients (15\%). IM and anti-TNF were required in 76/142 (54\%) and 44/142 (31\%), respectively (Figure 7). The median time to loss of mild disease status is illustrated in Table 5. The Kalpan-Meier curve of loss of mild UC is shown in Figure 8.

In univariate analysis, no demographic factors were associated with mild UC. The disease characteristics at 1 year associated with mild UC in the long term were the rectal location $(p=0.01$, proctitis vs. rectosigmoiditis: OR: $2.02,95 \% \mathrm{CI}=0.97-4.2$; proctitis vs. pancolitis: $\mathrm{OR}=3.85,95 \% \mathrm{CI}=1.6-9.3)$ and the absence of corticosteroids intake during the first year $(p=0.004$, OR $=3.32,95 \% \mathrm{CI}=1.46-7.58)$. No factor was significantly associated with mild UC in the multivariate analysis.

Among patients with mild UC 1 year after the diagnosis factors associated with the persistence of mild CD over the time were analysed. In univariate analysis, only older age at diagnosis was associated with the persistence of mild UC over the time ( $p=0.006, \mathrm{HR}=1.022,95 \% \mathrm{CI}=1.01-1.04)$ (Table 4).

\section{Discussion}

The present study suggests that about one-third of CD and three quarters of UC had a mild IBD defined 1 year after diagnosis. A few CD patients (12\%) still had a mild CD over the long term, while about $50 \%$ of UC patients still had a mild disease after a long follow-up period. In addition, the study shows that an older age at diagnostic was predictive of the persistence of a mild CD and UC over the time.

This is the first study proposing a definition of mild CD and mild UC and looking for its persistence over time. For both diseases, we used a strict definition including the absence of irreversible tissue damages: absence of stricture, fistulae, perianal lesions, surgery in $\mathrm{CD}$, absence of surgery in UC. In $\mathrm{CD}$, Pariente et al. [1] demonstrated that time could lead to complications and additive digestive damages. This accumulation is suspected to progressively impair the intestinal function and the quality of life.[8] Active chronic UC can also induce a loss of colonic function [2] and an increased risk of colonic cancer.[9] Our definition of mild CD and UC also excluded the requirement of IM or anti-TNF in the course of the disease because they are usually associated with a worsening of the disease and with an increased incidence of infectious [10-12] and oncologic complications.[13,14]

We described a 44\%-rate of UC patients having a persisting mild disease over the long term which is consistent with the previous data.[15,16] However, the $90 \%$ rate of non-mild CD at the maximum follow-up is probably largely overestimated. Two independent reference centre studies $[3,4]$ assessed the outcome of disabling CD at 5 years. The definition of disabling CD was close to the opposite of our mild disease definition. Only $20-40 \%$ of the patients did not have a disabling CD at 5 years compared to $17 \%$ in our cohort. In population-based studies, however, about $50 \%$ of the patients remained complications free at 5 years.[17] The initial 3 years following 
diagnosis were the more active and some degree of disease extinction was observed over time. In addition, few patients with a low disease activity during the first year experienced a significant worsening of the disease over time.[17] In our study, a large proportion of mild CD at 1 year finally did not meet the criteria of mild disease over the time. This is in contradiction with the concept of disease extinction suggested in previously published population-based study [17] and may indicate a recruitment bias: the patients with initial mild disease we recruited are precisely those who lost this mild disease status and were then referred to our centre.

The characteristics of the disease worsening must also be taken into account. At 5 years only $7 \%, 6 \%$ and $11 \%$ experienced strictures, fistulae, perianal lesions, respectively, and only 17\%, 28\% and 31\%, respectively, at the maximum follow-up (median 16 years). Except for perianal lesions,[18-20] the rates of stricturing and penetrating disease were lower in this population of mild CD at 1 year than in the global CD population previously described. According to our definition, a large proportion of patients lost the mild character of the disease because of the introduction of IM $(70 \%)$ or anti-TNF $(50 \%)$. This treatment strategy may reflect an aggressive course of the disease but also a strategy of optimised step-up based on close monitoring of the patients. An argument for this hypothesis is the increased incidence of non-mild disease in most recent patients, probably reflecting an evolution in the treatment strategy over the last two decades. Surgery had to be performed in $43 \%$ of the CD patients after more than 15 years of follow-up. Solberg et al. [17] reported 38\% of surgery after 10 years. The findings from our referral centre are close to the results of this Norwegian population-based cohort taking into account the longer follow-up in our cohort. This can reflect a strategy of optimised step-up treatment strategy leading to less complication rates and less surgery. Although similar, our results cannot be strictly compared with those from the IBSEN study because this is not a population-based study but a monocentre retrospective cohort. The population-based cohort of Rungoe et al. [21] demonstrated a decreased probability rate of surgery at 5 years in CD and UC in the last 10 years but without a surgery-sparing effect of newer medications. Due to the retrospective character of the study, the type of resection was not taken into account. A short ileal resection for stricturing disease can be associated with a favourable disease outcome, while a long ileal resection or any colonic resection is usually associated with severe disease and functional bowel impairment. Thus, some surgeries in our cohort can be associated with good clinical outcome despite our definition of non-mild disease in the present study. Few patients with UC underwent surgery in our study (15\%). Recent population-based studies reported that approximately $10 \%$ of patients with UC will require colectomy after 10 years.[22,23] A recent study reported a need of colectomy of $7.5 \%, 10.4 \%$ and $14.8 \%$, respectively, at 5 , 10 and 20 years after the diagnosis.[24] A significant number of colectomies occurred in the first year of the disease and during the first hospitalisation for UC. Elective colectomy rates have significantly decreased in the last decade and alongside the use of purine and anti-TNF increased in UC suggesting a better control of the disease with the medications.[24] In our cohort, $47 \%$ and $27 \%$ of the patients required finally IM and anti-TNF, respectively.

The existence of a mild CD 1 year after the diagnosis was associated with the absence of the use of corticosteroids and the use of 5ASA in the first year reflecting a milder disease course. The initial need for corticosteroids has already been identified as a predictor of a disabling and severe disease $[3,4]$ and as a predictor of complication in non-complicated CD at diagnosis.[25]

An older age at diagnosis was associated with a benign evolution of CD and with the persistence of mild CD in the group of patients having a mild CD at 1 year. Our findings confirm the results from the Saint-Antoine cohort in Paris [5] that demonstrated an association of this factor with mild to moderate CD. Consistently, the reverse was observed for young age at diagnosis, which has been associated with a severe course of the disease.[3,4] Disease location had no impact on the overall outcome of CD throughout the long-term follow-up of our cohort. This confirms again the Saint-Antoine results, while Loly et al. [4] found a significant association with more extensive $\mathrm{CD}$ and disabling disease.

We demonstrated that mild UC 1 year after the diagnosis and in the long term was associated with proctitis at 1 year. Lower rate of hospitalisation and corticosteroids use was associated with mild UC 1 year after the diagnosis, reflecting as in $\mathrm{CD}$ a milder disease course. The only factor associated with persistence of mild disease over time in the group of patients with mild UC at 1 year was an older age at diagnosis. This factor has already been associated with a less severe UC [22,26,27] but the issue of these studies was the need for colectomy. Our work confirms this mild outcome also taking into account the need for IM and anti-TNF.

Our study has several limitations. First because of the retrospective character of the study potentially associated with incomplete data and follow-up we only selected recently reviewed patients with complete follow-up of minimum 5 years. This can induce a bias by selecting more severe patient requiring a regular follow-up. Because of the retrospective character of the study we obtained incomplete data on the CS use in the first year and the hospitalisation rate during the first year and had to exclude them in the multivariate analysis although there were significant in the univariate analysis. Second, our population is a referral centre-based cohort probably including 
more severe patients with higher rates of complications, surgery and a higher need for IM and anti-TNF compared to population-based studies. This recruitment bias may underestimate the number of patients with mild IBD. In our cohort, the number of CD patients is higher than the number of UC patients reflecting a higher prevalence of CD than UC in our region.[28]. Third, due to the retrospective character of the study, the type of surgery was not taken into account. A short ileal resection for stricturing disease can be associated with a favourable disease outcome in opposite to long ileal resection or any colonic resection associated with severe disease and functional bowel impairment. Thus, some surgeries in our cohort can be associated with good clinical outcome despite our definition of non-mild disease in the present study.

In conclusion, only a small minority of CD patients but up to half of the UC patients followed up in a referral centre have persisting mild disease over time. Apart from an older age at diagnosis, no other factor was predictive of persisting mild CD and UC. This suggests a prudent step-up approach in older patients, while a close monitoring is probably the most appropriate option in younger patients, particularly in CD to avoid the overtreatment and reduce a significant economic burden due to biological and immunosuppressive therapy.

\section{Disclosure statement}

The authors report no conflicts of interest. The authors alone are responsible for the content and writing of the paper.

\section{References}

[1] Pariente B, Mary JY, Danese S, et al. Development of the Crohn's disease digestive damage score, the Lemann score. Inflamm Bowel Dis. 2011;17:1415-1422.

[2] Bernardini N, Segnani C, Ippolito C, et al. Immunohistochemical analysis of myenteric ganglia and interstitial cells of Cajal in ulcerative colitis. J Cell Mol Med. 2012;16:318-327.

[3] Beaugerie L, Seksik P, Nion-Larmurier I, et al. Predictors of Crohn's disease. Gastroenterology. 2006;130:650-665.

[4] Loly C, Belaiche J, Louis E. Predictors of severe Crohn's disease. Scand J Gastroenterol. 2008;43:948-954.

[5] Cosnes J, Bourrier A, Nion-Larmurier I, et al. Factors affecting outcomes in Crohn's disease over 15 years. Gut. 2012;61:1140-1145.

[6] Lennard-Jones JE. Classification of inflammatory bowel disease. Scand J Gastroenterol Suppl. 1989;170:2-6. discussion 16-19.

[7] Silverberg MS, Satsangi J, Ahmad T, et al. Toward an integrated clinical, molecular and serological classification of inflammatory bowel disease: report of a Working Party of the 2005 Montreal World Congress of Gastroenterology. Can J Gastroenterol. 2005;19:5-36.

[8] van der Have M, van der Aalst KS, Kaptein AA, et al. Determinants of health-related quality of life in Crohn's disease: a systematic review and meta-analysis. J Crohns Colitis. 2014;8:93-106.

[9] Jess T, Simonsen J, Jørgensen KT, et al. Decreasing risk of colorectal cancer in patients with inflammatory bowel disease over 30 years. Gastroenterology.2012;143:375-381.

[10] Colombel JF, Loftus EV, Tremaine WJ, et al. The safety profile of infliximab in patients with Crohn's disease: the Mayo clinic experience in 500 patients. Gastroenterology. 2004;126:19-31.

[11] Toruner M, Loftus EV, Harmsen WS, et al. Risk factors for opportunistic infections in patients with inflammatory bowel disease. Gastroenterology. 2008;134:929-936.

[12] Lichtenstein GR, Feagan BG, Cohen RD, et al. Serious infections and mortality in association with therapies for Crohn's disease: TREAT registry. Clin Gastroenterol Hepatol. 2006;4:621-630.

[13] Beaugerie L, Brousse N, Bouvier AM, et al. Lymphoproliferative disorders in patients receiving thiopurines for inflammatory bowel disease: a prospective observational cohort study. Lancet. 2009;374:1617-1625.

[14] Peyrin-Biroulet L, Khosrotehrani K, Carrat F, et al. Increased risk for nonmelanoma skin cancers in patients who receive thiopurines for inflammatory bowel disease. Gastroenterology. $2011 ; 141: 1621$-1628.

[15] Jess T, Rungoe C, Peyrin-Biroulet L. Risk of colorectal cancer in patients with ulcerative colitis: a metaanalysis of population-based cohort studies. Clin Gastroenterol Hepatol. 2012;10:639-645.

[16] Jess T, Frisch M, Simonsen J. Trends in overall and cause-specific mortality among patients with 
inflammatory bowel disease from 1982 to 2010. Clin Gastroenterol Hepatol. 2013;11:43-48.

[17] Solberg I, Vatn MH, Høie O, et al. Clinical course in Crohn's disease: results of a Norwegian populationbased ten year follow-up study. Clin Gastroenterol Hepatol. 2007;5:1430-1438.

[18] Hellers G, Bergstrand O, Ewerth S, et al. Occurrence and outcome after primary treatment of anal fistulae in Crohn's disease. Gut. 1980;21:525-527.

[19] Louis E, Collard A, Oger AF, et al. Behaviour of Crohn's disease according to the Vienna classification: changing pattern over the course of the disease. Gut. 2001;49:777-782.

[20] Cosnes J, Cattan S, Blain A, et al. Long-term evolution of disease behavior of Crohn's disease. Inflamm Bowel Dis. 2002;8:244-250.

[21] Rungoe C, Langholz E, Anderson M, et al. Changes in medical treatment and surgery rates in inflammatory bowel disease: a nationwide cohort study 1979-2011. Gut. 2014;63:1607-1616.

[22] Solberg IC, Lygren I, Jahnsen J, et al. Clinical course during the first 10 years of ulcerative colitis: results from a population-based inception cohort (IBSEN study). Scand J Gastroenterol. 2009;44:431-440.

[23] Höie O, Wolters F, Riis L, et al. Ulcerative colitis: patient characteristics may predict 10-yr disease recurrence in a European-wide population-based cohort. Am J Gastroenterol. 2007;102:1692-1701.

[24] Kaplan GG, Seow CH, Ghosh S, et al. Decreasing colectomy rates for ulcerative colitis: a population-based time trend study. Am J Gastroenterol. 2012; 107:1879-1887.

[25] Solberg IC, Cvancarova M, Vatn MH, et al. Risk matrix for prediction of advanced disease in a population based study of patients with Crohn's Disease (the IBSEN Study). Inflamm Bowel Dis. 2014;20:60-68.

[26] Ananthakrishnan AN, Issa M, Beaulieu DB, et al. History of medical hospitalization predicts future need for colectomy in patients with ulcerative colitis. Inflamm Bowel Dis. 2009;15:176-181.

[27] Sandborn WJ, Rutgeerts P, Feagan BG, et al. Colectomy comparison after treatment of ulcerative colitis with placebo or infliximab. Gastroenterology. 2009;137: 1250-1260.

[28] Latour P, Louis E, Belaiche J. Incidence of inflammatory bowel disease in the area of Liège: a 3 years prospective study (1993-1996). Acta Gastroenterol Belg. 1998;61:410-413. 